Review began 10/21/2021 Review ended 10/28/2021 Published 11/09/2021

๑) Copyright 2021

Diwan et al. This is an open access article distributed under the terms of the Creative Commons Attribution License CC-BY 4.0. which permits unrestricted use, distribution, and reproduction in any medium, provided the original author and source are credited.

\section{Impact and Outcomes of Regional Anesthesia Techniques in Elderly Patients With Fracture of Proximal Femur: A Retrospective Study}

Sandeep Diwan ${ }^{1}$, André Van Zundert ${ }^{2}$, Abhijit Nair ${ }^{3}$, Parag K. Sancheti ${ }^{4}$, Chetan Pradhan ${ }^{5}$, Chetan Puram ${ }^{5}$

1. Anaesthesiology, Sancheti Institute for Orthopaedics and Rehabilitation, Pune, IND 2. Anaesthesia and Perioperative Medicine, Royal Brisbane and Women's Hospital, Brisbane, AUS 3. Anesthesiology, Ibra Hospital, Ibra, OMN 4. Orthopaedics, Sancheti Institution for Orthopaedics and Rehabilitation, Pune, IND 5. Orthopaedics, Sancheti Institute for Orthopaedics and rehabilitation, Pune, IND

Corresponding author: Abhijit Nair, abhijitnair95@gmail.com

\section{Abstract}

\section{Background}

Although subarachnoid block (SAB) is the most popular regional anesthesia (RA) technique for fixation of femur fractures, continuous lumbar epidural (CLE) anesthesia, and lumbosacral plexus blocks (LSPB) are also employed in specific situations. The choice of RA technique depends on either the choice of the anesthesiologist or based on the underlying comorbidities. At our institute, we anesthetize elderly patients who come for fixation of femur fracture with multiple comorbidities using RA techniques as mentioned based on comorbidities and overall general condition.

\section{Methods}

In a cohort of 184 elderly patients, we analyzed RA techniques employed over a period of five years in elderly patients admitted with fractures of the proximal femur, its hemodynamic implications and thus attempted to find the suitable RA technique with minimal adverse events after ethics committee approval. We also compared the length of stay in the hospital in relation to RA techniques.

\section{Results}

The demographic data was comparable with no significant difference in administering the three RA techniques. SAB, CLE and LSPB was implemented at 33.33\%, 35.96\%, and 30.7\% respectively. Perioperative noradrenaline infusion was a feature in patients who received SAB $(p<0 / 001)$. The higher number of CLE and LSPB patients had a length of stay of fewer than 48 hours whereas most SAB patients had a length of stay of more than $48 \mathrm{hrs}(\mathrm{p}<0.001)$.

\section{Conclusion}

Elderly patients with multiple comorbidities should be offered CLE instead of SAB so as to maintain stable hemodynamics. RA technique in an elderly patient with multiple comorbidities should be standardized so as to provide uneventful surgical anesthesia.

Categories: Anesthesiology, Pain Management, Trauma

Keywords: regional anesthesia, geriatric, fracture, femur, lumbosacral plexus, epidural anesthesia, spinal anesthesia

\section{Introduction}

Elderly patients with comorbidities who sustain complex proximal femoral fractures (PFF) are often referred to specialized orthopedic surgery centers in view of associated comorbidities and the availability of definitive geriatric protocols [1]. No statistical significance was found in mortality between subarachnoid block (SAB) and general anesthesia techniques employed for hip fractures [2,3]. One of the most striking features of the management of PFF is the wide variation in the use of regional anesthesia (RA) techniques, though even in a well-established tertiary center, controversy and variations exist regarding various options of regional anesthesia (RA). SAB is the commonest form of RA utilized in most hospitals. In our singlecenter experience, we examined the outcomes related to different RA techniques in elderly patients with PFF. The primary aim was to evaluate the impact of various RA techniques employed, its case-specific distribution for elderly American Society of Anesthesiologists' physical status (ASA-PS) III and IV patients with PFF's, and their outcomes in the form of hemodynamic complications. The secondary aim was to evaluate the average length of stay in the hospital, in-hospital mortality on the fifth day (day of discharge) in relation to the RA techniques, specific complication associated with a RA technique, and propose standardization of RA techniques for PFFs. 


\section{Materials And Methods}

This single-center, observational study was approved by the institutional ethical committee (approved on 22nd November 2018). Medical records (preoperative, anesthesia, and postoperative nursing charts) obtained from the medical record department were examined for patients admitted with PFFs and who underwent a proximal femoral nail (PFN). The study population included 184 adults aged 70 to 97 and categorized in ASA-PS III and IV, between January 2015 to June 2018. Of 184 patients, 114 patients (50 male; 64 female) qualified for the study. In 70 patients, GA was administered in patients on oral anticoagulants or low molecular weight heparins, and inadequate RA qualified for the exclusion.

In our institution, with an INR less than 1.5, an activated partial thromboplastin time (aPTT) less than 35 seconds, platelet count of more than 80,000 , last dose of antiplatelet [clopidogrel] stopped for more than five days and last dose of low molecular weight heparin injection [enoxaparin] more than 12 hours are suitable candidates for neuraxial anesthesia. An unusual, prolonged aPTT was screened for deficient coagulation factors by the hematologist.

Based on the available information obtained from the charts the patients were assigned to one particular category of comorbid (example: underwent coronary artery bypass grafting, diabetes mellitus, coronary artery disease). Records were tracked for vital parameters, laboratory investigations, preoperative medications, $\mathrm{x}$-ray chest and spine, electrocardiogram and echocardiography, and another opinion from a specialist consultant if performed.

Information regarding preoperative counseling and patient informed consent was obtained from the anesthesia charts. The type of RA technique employed in each case and the details of the technique were noted. The type of RA implemented in a particular cohort of comorbidities was also noted. On arrival in the operating room (OR) after appropriately sized intravenous (IV) cannulation and application of essential monitors (pulse oximeter, non-invasive blood pressure cuff, electrocardiograph), all patients received ultrasound-guided femoral nerve block with $1 \%$ lidocaine $10 \mathrm{ml}$ in the supine position. SAB and continuous lumbar epidural (CLE) were administered in either sitting or lateral position and lumbosacral plexus block (LSPB) in the lateral position with operated side non-dependant.

SAB was administered using $0.5 \%$ bupivacaine $2-2.5 \mathrm{ml}$ with $150 \mu \mathrm{g}$ buprenorphine as an adjuvant. CLE was in the form of a continuous infusion through a $20 \mathrm{G}$ catheter at the L2-3 level. An initial injection of 8-10ml $0.5 \%$ bupivacaine followed by another bolus of $3-5 \mathrm{ml}$ of the same solution was administered through the catheter only if the level of surgical anesthesia was not adequate. Postoperatively all patients received $0.1 \%$ ropivacaine at $6 \mathrm{ml}$ / hour for 48 hours. For LSPB, a compound solution of $0.5 \%$ bupivacaine $2 \mathrm{mg} / \mathrm{kg}$ and $1.5 \%$ lidocaine $6 \mathrm{mg} / \mathrm{kg}$ with $1 \mu \mathrm{g} / \mathrm{kg}$ clonidine was used for LSPB achieved with neurostimulation [0.4mg.kg-1for lumbar plexus block (LPB) and $0.2 \mathrm{mg} / \mathrm{kg}$ for sacral plexus block (SPB)]. Quadriceps contractions at $0.4 \mathrm{~mA}$ and plantar/dorsiflexion at $0.4 \mathrm{~mA}$ were the endpoints for LPB and SPB respectively.

All surgeries were performed in the lateral decubitus position with operative site non-dependant without a fracture table, by one of three experienced orthopedic surgeons, practicing for 20 years. The RA techniques were performed by a single anesthesiologist with 25 years of experience in RA. Invasive monitoring in the form of an arterial line and a central venous catheter was performed in patients with low ejection fraction (less than 30\%) and in presence of regional wall motion abnormalities. Intravenous paracetamol $1 \mathrm{gm}$ was infused at skin closure and every eighth hour thereafter, as a part of multimodal regimen in all groups. IV rescue analgesic was in the form of fentanyl $10 \mu \mathrm{g} / \mathrm{ml}$, initiated at $2 \mathrm{ml} / \mathrm{hour}$ after a bolus of $0.5 \mu \mathrm{g} / \mathrm{kg}$.

All patients were transferred to the intensive care unit (ICU), and if hemodynamically stable and not requiring monitoring were admitted to the ward. Patients who required perioperative vasoactive drugs (noradrenaline infusion=NAD infusion, dopamine infusion=DOP) were noted and analyzed separately.

The continuous variables were summarised using mean+/- standard deviation or median (interquartile range) as necessary, and categorical variables by percentage or absolute numbers. The difference in mean between groups of types of anesthesia was analyzed by one-way analysis of variation (ANOVA). The association between categorical variables and type of anesthesia was determined by Fisher's exact test. SPSS Statistics v. 21 (IBM Corp., Armonk, NY) was used for statistical analysis. A p-value of less than 0.05 was considered statistically significant.

\section{Results}

Overall, 114 ASA-PS III and IV patients underwent surgery for PFF between January 2015 and June 2018. Of 114 patients, 77 had intertrochanteric fractures while 37 suffered from subtrochanteric fractures. Thirtyeight patients received a SAB, 41 patients a continuous CLE, and 35 patients were administered an LSPB (Table 1). 


\section{Cureus}

\begin{tabular}{|c|c|c|c|c|c|}
\hline & & $\operatorname{CLE}(n=41)$ & LSPB (n=35) & SAB $(n=38)$ & p-value \\
\hline \multicolumn{2}{|c|}{ Age in years Mean \pm standard deviation } & $77.39 \pm 1.43$ & $77.63 \pm 1.64$ & $80.39 \pm 1.54$ & $p=0.305$ \\
\hline \multirow{2}{*}{ Gender } & Male $\mathrm{n}(\%)$ & $18(36 \%)$ & $15(30 \%)$ & $17(34 \%)$ & \multirow{2}{*}{$p=0.987$} \\
\hline & Female n (\%) & $23(35.9 \%)$ & $20(31.3 \%)$ & $21(32.8 \%)$ & \\
\hline \multirow{4}{*}{ Fracture type } & IT left $n(\%)$ & $19(33.33 \%)$ & $20(35.1 \%)$ & $18(31.6 \%)$ & \multirow{4}{*}{$p=0.6$} \\
\hline & IT right $\mathrm{n}(\%)$ & $18(39.1 \%)$ & $12(26.1 \%)$ & $16(34.8 \%)$ & \\
\hline & ST left n (\%) & $2(33.3 \%)$ & $3(50 \%)$ & $1(16.7 \%)$ & \\
\hline & ST right $\mathrm{n}(\%)$ & $2(40 \%)$ & $0(0 \%)$ & $3(60 \%)$ & \\
\hline \multirow{2}{*}{ ASA-PS } & III & $17(28.8 \%)$ & $18(30.5 \%)$ & $24(40.7 \%)$ & \multirow{2}{*}{$p=0.156$} \\
\hline & IV & $24(43.6 \%)$ & $17(30.9 \%)$ & $14(25.5 \%)$ & \\
\hline
\end{tabular}

\section{TABLE 1: Demographic characteristics of participants}

CLE: continuous lumbar epidural; LSPB: lumbosacral plexus block; SAB: subarachnoid block, ASA-PS: American Society of Anesthesiologists'-Physical Status; IT: intertrochanteric; ST: subtrochanteric

Statistical significance: $p<0.05$

The median age was 80 years with an interquartile range of (74.5-80.28). In ASA-PS III and IV patients, a CLE was administered more often than LSPB, whereas SAB was utilized the least ( $\mathrm{p}<0.001$ ) (Table 2). 


\begin{tabular}{|c|c|c|c|c|c|}
\hline & & \multicolumn{3}{|c|}{ Type of anesthesia } & \multirow[b]{2}{*}{$\mathrm{p}$-value } \\
\hline & & CLE $(n=41)$ & LSPB $(n=35)$ & $\mathrm{SAB}(\mathrm{n}=38)$ & \\
\hline \multirow{2}{*}{ Cardiovascular } & $\mathrm{N}$ & $5(45.5 \%)$ & $5(45.5 \%)$ & $1(9.1 \%)$ & $p=0.163$ \\
\hline & $\mathrm{Y}$ & $36(35.0 \%)$ & $30(29.1 \%)$ & 37 (35.9\%) & \\
\hline \multirow{2}{*}{ CAD } & $\mathrm{N}$ & 15 (22.4\%) & $20(29.9 \%)$ & 32 (47.8\%) & \multirow{2}{*}{$p<0.001$} \\
\hline & Y & $26(55.3 \%$ & $15(31.9 \%)$ & $6(12.8 \%)$ & \\
\hline \multirow{2}{*}{ Respiratory } & $\mathrm{N}$ & 31 (34.1\%) & 28 (30.8\%) & 32 (35.2\%) & \multirow{2}{*}{$p=0.635$} \\
\hline & $\mathrm{Y}$ & 10 (43.5\%) & $7(30.4 \%)$ & $6(26.1 \%)$ & \\
\hline \multirow{2}{*}{ Endocrine } & $\mathrm{N}$ & $14(29.2 \%)$ & 17 (35.4\%) & $17(35.4 \%)$ & \multirow{2}{*}{$\mathrm{p}=0.419$} \\
\hline & $Y$ & $27(40.9 \%)$ & $18(27.3 \%)$ & $21(31.8 \%)$ & \\
\hline \multirow{2}{*}{ Renal } & $\mathrm{N}$ & $26(38.2 \%)$ & $24(35.3 \%)$ & $18(26.5 \%)$ & \multirow{2}{*}{$\mathrm{p}=0.151$} \\
\hline & $\mathrm{Y}$ & $15(32.6 \%)$ & $11(23.9 \%)$ & $20(43.5 \%)$ & \\
\hline \multirow{2}{*}{ CNS } & $\mathrm{N}$ & $28(36.8 \%)$ & $22(28.9 \%)$ & $26(34.2 \%)$ & \multirow{2}{*}{$p=0.848$} \\
\hline & $\mathrm{Y}$ & $13(34.2 \%)$ & $13(34.2 \%)$ & $12(31.6 \%)$ & \\
\hline \multirow{2}{*}{ Malignancy } & $\mathrm{N}$ & 37 (34.3\%) & 34 (31.5\%) & 37 (34.3\%) & \multirow{2}{*}{$p=0.368$} \\
\hline & $\mathrm{Y}$ & $4(66.7 \%)$ & $1(16.7 \%)$ & $1(16.7 \%)$ & \\
\hline \multirow{2}{*}{ Electrolyte } & $\mathrm{N}$ & $13(27.7 \%)$ & $14(29.8 \%)$ & $20(42.6 \%)$ & \multirow{2}{*}{$p=0.166$} \\
\hline & $Y$ & $28(41.85 \%)$ & $21(31.3 \%)$ & $18(26.9 \%)$ & \\
\hline \multirow{2}{*}{ Spine instrumentation, metastasis } & $\mathrm{N}$ & $41(48.80 \%)$ & $5(5.95 \%)$ & $38(45.23 \%)$ & \multirow{2}{*}{$p<0.00001$} \\
\hline & $\mathrm{Y}$ & $0(0)$ & $30(100 \%))$ & $0(0)$ & \\
\hline \multirow{2}{*}{ NAD } & $\mathrm{N}$ & 40 (40.4\%) & $33(33.3 \%)$ & $26(26.3 \%)$ & \multirow{2}{*}{$p<0.001$} \\
\hline & Y & $1(6.7 \%)$ & $2(13.3 \%)$ & $12(80 \%)$ & \\
\hline \multirow{2}{*}{ Mortality } & $\mathrm{N}$ & $40(36.7 \%)$ & $33(30.3 \%)$ & 36 (33\%) & \multirow{2}{*}{$\mathrm{p}=0.745$} \\
\hline & $\mathrm{Y}$ & $1(20 \%)$ & $2(40 \%)$ & $2(40 \%)$ & \\
\hline \multirow{4}{*}{ Cause of mortality } & $\mathrm{N}$ & $40(36.7 \%)$ & $33(30.3 \%)$ & 36 (33\%) & \multirow{4}{*}{$\mathrm{p}=0.258$} \\
\hline & ACS & $1(50 \%)$ & $1(50 \%)$ & $0(0 \%)$ & \\
\hline & AMI & $0(0 \%)$ & $1(100 \%)$ & $0(0 \%)$ & \\
\hline & PE & $0(0 \%)$ & $0(0 \%)$ & $2(100 \%)$ & \\
\hline
\end{tabular}

\section{TABLE 2: Association of comorbidities and adverse events with type of anesthesia}

NAD: noradrenaline; Y: yes; N: no; CNS: central nervous system; CAD: coronary artery disease; CLE: continuous lumbar epidural; LSPB: lumbar and sacral plexus block; SAB: subarachnoid block

The comorbidities were comparable for all three RA approaches except in patients with coronary artery disease $(\mathrm{CAD})(\mathrm{p}<0.01)$ who received CLE. In patients with spine pathologies and instrumentation, all received an LSPB ( $p=0.002735$ ) but considering the sample size, no statistical significance could be derived when compared to the other two techniques (Table 2). Hemodynamic was well preserved in all patients in whom a CLE or an LSPB was administered. In 38 patients who received a SAB (46.15\%), NORAD infusion was used intraoperatively and, in the ICU, $(\mathrm{p}<0.001)$. The length of stay was significantly associated with the type of anesthesia. A higher number of CLE and LSB patients had a length of stay of 24 hrs and less than 48 hrs, whereas most SAB patients had a length of stay of more than $48 \mathrm{hrs}(\mathrm{p}<0.001$, Table 3). In-hospital mortality was at $2.8 \%$ and $5.2 \%$ in patients who received LSPB and SAB, and none in whom CLE was administered. The overall mortality stood at $2.63 \%$. 


\section{Cureus}

\begin{tabular}{|c|c|c|c|c|}
\hline \multirow{2}{*}{ Length of stay } & \multicolumn{3}{|c|}{ Type of anesthesia } & \multirow[t]{2}{*}{$p$-value } \\
\hline & $\operatorname{CLE}(n=41)$ & $\operatorname{LSPB}(n=35)$ & $\mathrm{SAB}(\mathrm{n}=38)$ & \\
\hline $24 \mathrm{hr}$ & $24(54.5 \%)$ & $20(45.5 \%)$ & $0(0.0 \%)$ & $<0.00001$ \\
\hline $48 \mathrm{hr}$ & $12(28.6 \%)$ & $9(21.4 \%)$ & $21(50.0 \%)$ & \\
\hline $72 \mathrm{hr}$ & $0(0.0 \%)$ & $0(0.0 \%$ & $10(100.0 \%)$ & \\
\hline $96 \mathrm{hr}$ & $3(23.1 \%)$ & $4(30.8 \%$ & $6(46.2 \%)$ & \\
\hline $120 \mathrm{hr}$ & $2(40.0 \%)$ & $240.0 \%$ & $1(20.0 \%)$ & \\
\hline
\end{tabular}

\section{TABLE 3: Association of the length of stay in ICU with the type of anesthesia}

CLE: continuous lumbar epidural; LSPB: lumbar and sacral plexus block; SAB: subarachnoid block

\section{Discussion}

Our aim was to understand the impact of different types of RA techniques implemented in PFF undergoing a PFN, and outcomes in the form of whether there was an inclination of utilizing these techniques in a particular cohort with comorbidities and hemodynamic complications. Epidural was consistently effective in all ASA-PS III and IV, for anesthesia and analgesia purposes, and patients were hemodynamically stable throughout the perioperative course in patients with CAD. LSPB was administered particularly in the operated spine and metastatic spine disorders. SAB was associated with increased use of IV vasoactive drugs in the postoperative period.

Elderly patients with comorbidities sustaining complex PFFs require an early optimization and surgical intervention. Compared to general anesthesia, RA is associated with less incidence of in-hospital mortality and respiratory-related morbidity [4]. The type of a safe and feasible RA remains undefined and inconclusive in a cohort of PFFs. SAB was the only technique that is described, but discussion on other options like CLE and LSPB remains elusive. The Anesthesia Sprint Audit of Practice (ASAP) reports that the conduct of anesthesia appears to affect outcomes after hip fracture surgery [5]. With a mean arterial blood pressure (ABP) of less than $55 \mathrm{mmHg}$ for more than 20 minutes, a graded increase in the risk of renal and myocardial injury was noted [6]. In spite of these problems, anaesthesiologists continue to use SAB for PFFs without understanding the altered pathophysiology it creates in highly comorbid and frail patients [7]. Based on our study with a limited sample size, we recommend avoiding SAB in elderly patients with cardiac ailments (EF less than $30 \%$, presence of regional wall motion abnormality in echocardiography, long-standing diabetes with cardiomyopathy). The CLE is associated with a limited sympathectomy as against the extensive sympathectomy which is characteristic of SAB. We recommend incremental epidural anesthesia to have stable hemodynamic in the perioperative period. Further LA top-ups in epidural space allow extension of surgical anesthesia and postoperative pain relief through infusion pumps. Improved outcomes are directly related to the use of CLE [8].

Neuraxial techniques are challenging in patients with operated spine instrumentation [9]. Further neurological adverse events are known in patients with metastatic spine undergoing a SAB [10]. Not everyone is trained to deliver high-quality anesthesia using advanced nerve blocks in geriatric patients with PFFs. The LSPB are advanced deep blocks [11-13]. The risk profile of LPB is high as it could lead to epidural diffusion, potential local anesthesia toxicity, and lumbar plexopathy [14]. In view of advanced block and high-risk profiles, we recommend that LSPBs should be performed by experts. Although ultrasound provides a predictable paravertebral sonoanatomy for lumbar plexus and of sacral plexus blocks in adults, the same is not true in elderly patients [15]. A study by de Visme et al. mentions hypotension after SAB in patients over the age of 85 years was of larger magnitude and sustained for a long time [16]. Invasive cardiac monitoring using arterial and central line during an LSPB for hip surgery observed a significant drop in the MAP though there was no change in the cardiac index, ABP, and heart rate [17]. The in-hospital mortality was selected as the secondary study endpoint because of its probable immediate co-relation in the postoperative period. The 30th day and one-year mortality are never affected by the type of anesthesia used during surgery. However, in our series, RA techniques were employed in ASA-PS III and IV patients, and there was no mortality in patients who received CLE but it was $2.8 \%$ and $5.2 \%$ of patients who were administered LSPB and SAB respectively, with overall mortality at $2.63 \%$. Incremental epidural analgesia can be achieved with titrated doses of local anesthetic injected in the lumbar epidural space. In our cases who received segmental CLE, patients with severe $\mathrm{CAD}$ and respiratory disorders had better outcomes.

The sample size was a major limitation. Confounders (patients were heterogeneous with multiple comorbidities present in the same patient) and selection bias are limitations in an observational study; however, a randomized controlled trial is unfeasible for a similar study. Interventions in the observational 


\section{Conclusions}

Elderly patients with significant comorbidities should be offered CLE instead of SAB so as to maintain stable hemodynamics. RA technique in elderly patients with multiple comorbidities should be standardized so as to provide uneventful surgical anesthesia. Elderly patients undergoing the surgical procedure are susceptible to postoperative cognitive dysfunction and delirium, secondary to higher pain scores and opioid levels.

However, continuous epidural analgesia benefits in preventing such occurrences, leading to an early hospital discharge. We recommend CLE anesthesia in elderly patients with ASA-PS III and IV. An LSPB should be preferred in instrumented and pathological spines. SAB should be sparingly administered in these patients given the unstable perioperative hemodynamics and an increased ICU stay.

\section{Additional Information \\ Disclosures}

Human subjects: Consent was obtained or waived by all participants in this study. Institutional Ethics Committee, Sancheti Institute of Orthopedics and Rehabilitation, Pune-411005 issued approval IEC/2018/22. Animal subjects: All authors have confirmed that this study did not involve animal subjects or tissue. Conflicts of interest: In compliance with the ICMJE uniform disclosure form, all authors declare the following: Payment/services info: All authors have declared that no financial support was received from any organization for the submitted work. Financial relationships: All authors have declared that they have no financial relationships at present or within the previous three years with any organizations that might have an interest in the submitted work. Other relationships: All authors have declared that there are no other relationships or activities that could appear to have influenced the submitted work.

\section{Acknowledgements}

We wish to acknowledge Dr. Avinash SS, Associate Professor, Department of Biochemistry, Father Muller Hospital, Bengaluru, Karnataka State, India for helping us in performing and analyzing statistical tests for this study.

\section{References}

1. Mittal R, Banerjee S: Proximal femoral fractures: Principles of management and review of literature . J Clin Orthop Trauma. 2012, 3:15-23. 10.1016/j.jcot.2012.04.001

2. Lončarić-Katušin M, Mišković $P$, Lavrnja-Skolan V, Katušin J, Bakota B, Žunić J: General versus spinal anaesthesia in proximal femoral fracture surgery - treatment outcomes. Injury. 2017, 48:S51-5. 10.1016/S0020-1383(17)30740-4

3. Brox WT, Chan PH, Cafri G, Inacio MC: Similar mortality with general or regional anesthesia in elderly hip fracture patients. Acta Orthop. 2016, 87:152-7. 10.3109/17453674.2015.1128781

4. Neuman MD, Silber JH, Elkassabany NM, Ludwig JM, Fleisher LA: Comparative effectiveness of regional versus general anesthesia for hip fracture surgery in adults. Anesthesiology. 2012, 117:72-92. 10.1097/ALN.0b013e3182545e7c

5. White SM, Moppett IK, Griffiths R, et al.: Secondary analysis of outcomes after 11,085 hip fracture operations from the prospective UK Anaesthesia Sprint Audit of Practice (ASAP-2). Anaesthesia. 2016, 71:506-14. 10.1111/anae.13415

6. Walsh M, Devereaux PJ, Garg AX, et al.: Relationship between intraoperative mean arterial pressure and clinical outcomes after noncardiac surgery: toward an empirical definition of hypotension. Anesthesiology. 2013, 119:507-15. 10.1097/ALN.0b013e3182a10e26

7. Griffiths R, Alper J, Beckingsale A, et al.: Management of proximal femoral fractures 2011: Association of Anaesthetists of Great Britain and Ireland. Anaesthesia. 2012, 67:85-98. 10.1111/j.1365-2044.2011.06957.x

8. Pöpping DM, Elia N, Van Aken HK, et al.: Impact of epidural analgesia on mortality and morbidity after surgery: systematic review and meta-analysis of randomized controlled trials. Ann Surg. 2014, 259:1056-67. 10.1097/SLA.0000000000000237

9. Sharma M, McConachie: Neuraxial blocks in parturients with scoliosis and after spinal surgery . J Obstet Anaesth Crit Care. 2016, 6:70-4. 10.4103/2249-4472.191594

10. Cherng YG, Chen IY, Liu FL, Wang MH: Paraplegia following spinal anesthesia in a patient with an undiagnosed metastatic spinal tumor. Acta Anaesthesiol Taiwan. 2008, 46:86-90. 10.1016/S18754597(08)60033-8

11. Amiri HR, Zamani MM, Safari S: Lumbar plexus block for management of hip surgeries . Anesth Pain Med. 2014, 4:19407. 10.5704/MOJ.1511.004

12. Petchara S, Paphon S, Vanlapa A, Boontikar P, Disya K: Combined lumbar-sacral plexus block in high surgical risk geriatric patients undergoing early hip fracture surgery. Malays Orthop J. 2015, 9:28-34. 10.5704/MOJ.1511.004

13. Sarkar Mitra T, Mukherjee A, Agarwal G, Rupert E: Lumbar plexus block: Safe anesthesia for hip surgery . Anesth Essays Res. 2012, 6:241-3. 10.4103/0259-1162.108356

14. Breslin DS, Martin G, Macleod DB, D'ercole F, Grant SA: Central nervous system toxicity following the administration of levobupivacaine for lumbar plexus block: A report of two cases. Reg Anesth Pain Med. 2003, 28:144-7.10.1053/rapm.2003.50127

15. Diwan S, Nair A, Dadke M, Sancheti P: Intricacies of ultrasound-guided lumbar plexus block in octogenarians: A retrospective case series [IN PRESS]. J Med Ultrasound. 2021, 


\section{Cureus}

16. de Visme V, Picart F, Le Jouan R, Legrand A, Savry C, Morin V: Combined lumbar and sacral plexus block compared with plain bupivacaine spinal anesthesia for hip fractures in the elderly. Reg Anesth Pain Med. 2000, 25:158-62. 10.1053/rapm.2000.0250158

17. de Leeuw MA, Slagt C, Hoeksema M, Zuurmond WW, Perez RS: Hemodynamic changes during a combined psoas compartment-sciatic nerve block for elective orthopedic surgery. Anesth Analg. 2011, 112:719-24. 10.1213/ANE.0b013e318206bc30

18. Yang W, Zilov A, Soewondo P, Bech OM, Sekkal F, Home PD: Observational studies: going beyond the boundaries of randomized controlled trials. Diabetes Res Clin Pract. 2010, 88 Suppl 1:S3-9. 10.1016/S01688227(10)70002-4 